\title{
Erratum to "Bernstein-type Theorems in Hypersurfaces with Constant Mean Curvature" [An Acad Bras Cienc 72(2000): 301-310]
}

\author{
MANFREDO P. DO CARMO ${ }^{1}$ and DETANG ZHOU ${ }^{2,3}$ \\ ${ }^{1}$ IMPA, Estrada Dona Castorina, 110-Jardim Botanico 22460-320 Rio de Janeiro, Brazil, \\ ${ }^{2}$ Department of Mathematics, Shandong University, Jinan, Shandong 250100, China \\ ${ }^{3}$ Departamento de Geometria, Universidade Federal Fluminense (UFF), 24020-140 Niterói, RJ, Brazil
}

Manuscript received on July 24, 2001; accepted for publication on August 1, 2001.

\begin{abstract}
An erratum to Lemma 2.1 in Do Carmo and Zhou (2000) is presented.

Key words: Riemannian manifold, eigenvalue, hypersurface, mean curvature.
\end{abstract}

\section{ERRATUM}

Replace Section 2 in Do Carmo and Zhou (2000) by the following. The resulting change in the lemma will not affect the rest of the paper.

\section{A RESULT ON NODAL DOMAINS}

In this section we prove a result on the nodal domains of $|\phi|$ which will be needed in our proof of main theorems. We first need to recall the definition of nodal domains.

Definition. An open domain $D$ is called the nodal domain of a function $f$ if $f(x) \neq 0$ for $x \in$ int $D$ and vanishes on the boundary of $\partial D$. We denote by $N(f)$ the number of disjoint bounded nodal domains of $f$.

Now we have the following lemma which follows directly from Proposition 2.2 below. We want to thank the referee who provided the clearer proof of Proposition 2.2.

Lemma 2.1. Let $M$ be a hypersurface in $R^{n+1}$ with constant mean curvature $H$. Then

$$
\operatorname{ind}(M) \geq N(|\phi|) .
$$

E-mail: manfredo@impa.br / zhou@impa.br 
Proof. Let $N=N(|\phi|)$ and $D_{1}, D_{2}, \cdots, D_{N}$ be the $N$ nodal domains of $|\phi|$ and let

$$
\varphi(u)=u^{2}+\frac{n(n-2)}{\sqrt{n(n-1)}} H u-n H^{2} .
$$

Then from (1.5) and Proposition 2.2 below we have functions $f_{1}, f_{2}, \cdots, f_{N}$ with supports in $D_{1}$, $D_{2}, \cdots, D_{N}$ respectively such that

$$
I\left(f_{i}, f_{i}\right)=\int_{D_{i}}\left(\left|\nabla f_{i}\right|^{2}-\varphi(u) f_{i}^{2}\right)<0 .
$$

Denote $W$ the linear subspace spanned by $f_{1}, f_{2}, \cdots, f_{N}$. Since they have disjoint supports, they are orthogonal and thus the dimension of $W$ is $N$. The index form $I(\cdot, \cdot)$ is negative definite on $W$ so the Morse index is greater than or equal to $N$.

Proposition 2.2. Let $(M, g)$ be Riemannian manifold and $u \geq 0$ be a continuous function satisfying the following inequality of Simons' type in the distribution sense

$$
u^{2} \varphi(u) \geq a|\nabla u|_{g}^{2}-u \Delta_{g} u
$$

where $a>0$ is a constant and $\varphi$ is a continuous function on $R$. If $u$ has a relatively compact nodal domain $D$, then there exists a function $f_{D}$ with support in $D$ such that

$$
\int_{D}\left(|\nabla f|^{2}-\varphi(u) f^{2}\right)<0 .
$$

Proof. Suppose that $u$ admits a relatively compact nodal domain $D$. Write $q:=\varphi(u)$ and $v:=\log u$ on $D$. Thus (2.2) can be written as

$$
q \geq a|\nabla v|_{g}^{2}-\Delta_{g} v-|\nabla v|_{g}^{2}
$$

Then for any Lipschitz function $f$ with support in $D$ and vanishing at $\partial D$, we have

$$
\int_{D}\left(|\nabla f|^{2}-q f^{2}\right) \leq-a \int_{D} f^{2}|\nabla v|^{2}+\int_{D}|\nabla f-f \nabla v|^{2} .
$$

Let $f=w u$, for some function $w$ to be determined. We obtain

$$
\int_{D}\left(|\nabla f|^{2}-q f^{2}\right) \leq-a \int_{D} w^{2}|\nabla u|^{2}+\int_{D} u^{2}|\nabla w|^{2} .
$$

For all $b$ such that $U / 2 \leq b \leq U$, where $U:=\sup _{D} u$, set

$$
w_{b}(x)= \begin{cases}b & \text { as } u(x) \leq b \\ u(x), & \text { as } u(x)>b\end{cases}
$$


Denote $D_{+}$(resp. $\left.D_{-}\right)$the set of points in $D$ with $u(x) \geq b$ (resp. $\left.u(x) \leq b\right)$. A simple calculation leads to:

$$
\int_{D}\left(|\nabla f|^{2}-q f^{2}\right) \leq \int_{D_{+}} u^{2}|\nabla u|^{2}-\frac{a U^{2}}{4} \int_{D}|\nabla u|^{2} .
$$

When $b$ goes to $U$, the first term of right hand side tends to 0 (because $|\nabla u|^{2}$ is integrable), while the second term is fixed. It follows that $\int_{D}\left(|\nabla f|^{2}-q f^{2}\right)<0$ for all functions $f=w_{b} u$, when $b$ is close to $U$. The conclusion is proved.

\section{REFERENCE}

Do Carmo MP and Zhou D. 2000. Bernstein-type Theorems in Hypersurfaces with Constant Mean Curvature. An Acad Bras Cienc 72: 301-310. 